DESENVOLVIMENTO ECONÔMICO E REGIONAL

\title{
Desenvolvimento financeiro e crescimento econômico no Brasil (1995-2004) ${ }^{1}$
}

\author{
Fabrício J. Missio* \\ Frederico G. Jayme Jr.** \\ Ana Maria H. C. de Oliveira ${ }^{* * *}$
}

RESUMO: Este trabalho tem por objetivo analisar empiricamente a relação entre desenvolvimento financeiro e crescimento econômico para as Unidades Federativas do Brasil. Para tanto, realizou-se um exercício econométrico com o auxílio da técnica de regressão quantílica, o que permitiu um mapeamento mais completo do impacto gerado pelas medidas de desenvolvimento financeiro utilizadas sobre a variável resposta (PIB estadual). As evidências encontradas sugerem uma relação positiva entre desenvolvimento do sistema financeiro e crescimento econômico.

Palavras-chave: Intermediação financeira. Crescimento econômico. Regressão quantílica.

\section{INTRODUÇÃO}

No plano teórico, as interpretações sobre a influência do sistema financeiro para o crescimento são variadas e, em grande medida, controversas. A interpretação original de Shumpeter (1911), por exemplo, destaca o papel do financiamento na dinâmica das economias capitalistas, uma vez que deste depende o processo de inovações. Já a abordagem keynesiana reconhece a importância do setor financeiro ao entender como se dá a fragilidade do sistema capitalista. Neste caso, a importância do sistema financeiro advém do descasamento entre o financiamento e o retorno do investimento.

\footnotetext{
${ }^{1}$ Este trabalho é uma versão adaptada a este boletim de um trabalho mais completo que foi apresentado no Encontro Nacional da ANPEC de 2009.

* Professor de economia da Universidade Estadual de Mato Grosso do Sul (UEMS), doutorando em economia pelo Centro de Desenvolvimento e Planejamento Regional de Minas Gerais (Cedeplar) e bolsista Fundação de Apoio ao Desenvolvimento do Ensino, Ciência e Tecnologia do Estado de Mato Grosso do Sul (Fundect). Endereço eletrônico: fabriciomissio@gmail.com.

** Professor associado do Centro de Desenvolvimento e Planejamento Regional de Minas Gerais / Universidade Federal de Minas Gerais (Cedeplar/UFMG) e bolsista do Conselho Nacional de Desenvolvimento Científico e Tecnológico (CNPq). Endereço eletrônico: gonzaga@cedeplar.ufmg.br. Este autor gostaria de agradecer o financiamento do Cnpq e da Fundação de Amparo à Pesquisa do Estado de Minas Gerais (FAPEMIG).

*** Professora adjunta do Centro de Desenvolvimento e Planejamento Regional de Minas Gerais / Universidade Federal de Minas Gerais (Cedeplar/UFMG) e bolsista do Conselho Nacional de Desenvolvimento Científico e Tecnológico (Cnpq): Endereço eletrônico: ahermeto@cedeplar.ufmg.br
} 
Mais especificamente, a argumentação fundamenta-se no fato de que os agentes tendem a tomar seus recursos junto ao sistema financeiro por períodos curtos de tempo a uma dada taxa de juros. No entanto, como o investimento é uma atividade que oferece retornos de longo prazo, os agentes têm que buscar formas de refinanciamento ou de rolar suas dívidas. É exatamente nesse ponto que desempenha importância fundamental o sistema financeiro. Em um cenário onde as condições econômicas tenham se deteriorado e/ou onde a intermediação financeira seja pouco desenvolvida, o processo de refinanciamento das dívidas ou de alongamento destes empréstimos (mesmo por meio de taxas mais elevadas) pode ser inviabilizado, o que desestimula o investimento. Assim, quando não existem mecanismos que garantam este processo, apenas o animal spirits pode justificar o ato de investir. Por outro lado, quando o sistema financeiro é bem desenvolvido, há uma maior diversificação de riscos e maior oferta de crédito, o que acaba por afetar as expectativas dos empresários em relação à possibilidade de refinanciamento dos investimentos, criando um ambiente positivo para a realização do mesmo.

Do ponto de vista empírico, recentes trabalhos buscam quantificar esta relação no plano internacional, nacional ou regional. São exemplos destes, os trabalhos de King e Levine (1993), Rousseau e Wachtel (1998), Levine (1997), Demetriades e Arestis (1997), Levine, Loayza e Beck (2000), entre muitos outros, que em sua maioria confirmam a hipótese de que o desenvolvimento do sistema financeiro é um importante determinante da taxa de crescimento econômico.

Com base no que foi exposto, o presente trabalho tem por objetivo realizar um exercício econométrico a partir dos dados para os 26 Estados brasileiros e o Distrito Federal, com o auxílio da técnica de regressão quantílica, o que permite um mapeamento mais completo do impacto gerado pelas medidas de desenvolvimento financeiro utilizadas sobre a variável resposta (PIB estadual). O trabalho encontra-se dividido em duas seções, além dessa introdução e das considerações finais. A segunda seção apresenta a metodologia e a base de dados a ser empregada nos exercícios econométricos e a terceira seção apresenta os resultados alcançados.

\section{METODOLOGIA ECONOMÉTRICA E BASE DE DADOS}

Para o propósito deste trabalho, utiliza-se a técnica de regressão quantílica para dados empilhados ao longo do tempo, a qual permite analisar a associação existente entre a variável resposta (PIB estadual) com as variáveis explicativas (medidas de desenvolvimento

66 
financeiro) nos diversos quantis da distribuição condicional. Assim, a partir dessa metodologia é possível obter um mapeamento mais completo do impacto do desenvolvimento financeiro sobre o crescimento econômico, pois pode-se investigar como cada quantil responde, os invés de obter-se uma única reta de regressão.

A forma geral da equação estimada nesse trabalho é dada a seguir, onde a mesma representa o modelo de crescimento para dados em painel:

$$
g_{y i t}=\beta_{0}(\theta)+\beta_{1}(\theta) \log (D F)+\beta_{2}(\theta) \log Z_{i t}+\alpha_{i \theta}+\mu_{t \theta}+e_{i(\theta) t(\theta)}
$$

A variável dependente é o PIB (nominal, em logaritmo) do estado $i$ no período analisado; DF é a variável que representa o Desenvolvimento do Sistema Financeiro; Z são as variáveis de controle; $\alpha_{i \theta}$ é o efeito fixo de cada região e $\mu_{t \theta}$ é o efeito específico de tempo ${ }^{2}$. Deve-se ressaltar que o objetivo é verificar se o desenvolvimento financeiro aumenta o nível de renda nos estados brasileiros. Em termos teóricos, deve-se observar que as conclusões obtidas para o crescimento econômico têm implicações equivalentes sobre o nível de renda da economia.

Para testar o modelo será adotado, em primeiro lugar, uma estratégia semelhante a de King \& Levine (1993). Para tanto, a análise empírica se limitará a explorar o impacto de duas variáveis financeiras (como proxy para crédito) sobre o crescimento econômico, sendo estas: (ii) depósitos à vista (depvista), (ii) depósitos a prazo (deprazo). A segunda estratégia busca captar o desenvolvimento do sistema financeiro a partir do desenvolvimento de dois indicadores: tamanho do setor e o nível de atividade. Especificamente, os indicadores utilizados foram: i) Quanto ao tamanho do mercado a proxy de mensuração foi: passivo exigível (soma dos depósitos à vista e a prazo) sobre PIB (TamSB); ii) Quanto ao nível de atividade no mercado: crédito do sistema financeiro sobre o PIB (NivSB1) e empréstimos sobre o PIB (NivSB2).

Além disso, realizou-se o exercício econométrico que busca captar o argumento póskeynesiano. Em síntese, a análise pós-keynesiana se distingue das demais por abordar tanto o lado da oferta quanto o lado da demanda no mercado de crédito. A distinção está no fato de que, para estes autores, a oferta e a demanda de crédito são interdependentes e afetadas pela preferência pela liquidez, vinculada às expectativas que os agentes formam em um ambiente de incerteza. Assim, pelo lado da demanda por crédito, a preferência pela liquidez do público afetará suas respectivas definições de portfólio. Ou seja, quanto maior a preferência pela

\footnotetext{
2 Para captar o efeito fixo e o efeito específico de tempo foram utilizadas variáveis dummies que, para fins de simplificação, não serão reportadas.
} 
liquidez, maior as posições em ativos líquidos destes agentes e menor sua demanda por crédito. Nesse caso, a fim de captar este efeito utilizou-se o seguinte indicador ${ }^{3}$ : iii) preferência pela liquidez do público (PLP), que é dado pela razão entre os depósitos à vista e os depósitos totais.

O resultado esperado das estimações é de que quanto maior for o desenvolvimento do sistema financeiro (representado pelas variáveis financeiras e pelos indicadores construídos) maior deve ser o nível de renda dos estados. Ou seja, espera-se uma relação positiva entre essas duas variáveis. Exceção é feita em relação aos resultados do último exercício econométrico, porque quanto maior for a PLP, menor tende ser a oferta de crédito e, assim, o nível de renda.

Estabeleceram-se alguns controles utilizados usualmente na literatura. Estes controles envolvem a renda do período inicial (renda) para mensurar o efeito da convergência; o número de anos de estudo da população acima de 25 anos (educ) como proxy de qualificação, importante para entender desenvolvimento tecnológico; o grau de abertura de cada estado (calculado como a soma das exportações mais importações sobre o PIB); uma variável que representam gastos do governo como proporção do PIB em educação e cultura (educul), outra em saúde e saneamento (sausan) ${ }^{4}$ e outra na esfera jurídica (júri).

A escolha desse conjunto de variáveis de controle se justifica por serem estas correspondentes ou proxy para as variáveis que são amplamente utilizadas em trabalhos empíricos. A dificuldade, todavia, é que como a unidade de análise são as Unidades Federativas, a série para os dados disponíveis é reduzida, além de a mesma ser construída a partir de diferentes fontes. Os dados utilizados correspondem ao período de 1995 a 2004 e foram obtidos das seguintes fontes: a) variáveis financeiras - Cedeplar (LEMTe), a partir de dados do Banco Central do Brasil ${ }^{5}$; b) valor das exportações e importações - Ministério do Desenvolvimento, Indústria e Comércio Exterior; e demais variáveis no Ipeadata.

Do ponto de vista teórico e econométrico, a principal dificuldade do modelo a ser estimado é o problema da presença da endogeneidade. Essa endogeneidade advém de alguma variável omitida que pode estar presente no termo de erro e que é correlacionada com alguma variável explicativa. Para o caso específico do trabalho, a endogeneidade pode estar presente

\footnotetext{
${ }^{3}$ Para uma justificativa sobre a construção desses indicadores, ver Cavalcante, Crocco e Jayme Jr. (2006).

${ }^{4}$ Essa variável busca captar se uma melhor "saúde" dos trabalhadores tem efeitos positivos sobre o crescimento, como sugere a evidência empírica recente. Ver, entre outros, Barro (1996) e Bloom, D. E; Canning, D. e Sevilla, J. (2001).

${ }^{5}$ Laboratório de estudos sobre Moeda e Território (LEMTe) do Centro de Desenvolvimento e Planejamento Regional de Minas Gerais / Universidade Federal de Minas Gerais (Cedeplar/UFMG).
} 
na medida em que as variáveis que representam instituições não são consideradas explicitamente.

Existe consenso na literatura que "boas" instituições são favoráveis ao crescimento (Acemoglu, 2009). Todavia, esta literatura enfrenta no mínimo dois problemas: o primeiro, de ordem empírica, refere-se à definição de instrumentos e/ou proxies apropriadas para a qualidade das instituições (o que, de fato, são boas instituições?); e o segundo, do ponto de vista teórico, remete-se ao desafio de explicar as diferenças de crescimento de um determinado pais ou região sujeitos ao mesmo marco regulatório.

Mais especificamente, focando o objetivo deste trabalho, como seria possível explicar as disparidades entre as trajetórias de desenvolvimento no Brasil, uma vez que o arcabouço macro-institucional é constante? Segundo Naritomi (2007), para entender os padrões observados dentro do país, é preciso investigá-las na esfera local, a partir de dimensões mais concretas do que as que se tem discutido na literatura. Ao mesmo tempo, devido à constância das macro-instituições no território e ao caráter da federação brasileira, é necessário avançar no entendimento das diferenças entre os papéis de instituições de facto e de jure, i.e., entre o funcionamento efetivo das instituições e o que está formalmente estabelecido. Em outros termos, defende-se que, apesar da existência de um corpo legal em sua maior parte comum entre as regiões brasileiras, as instituições informais variam entre os estados, ou seja, as instituições atuais são endógenas, pois derivam da própria trajetória de desenvolvimento das regiões. Além disso, deve-se ressaltar que a literatura para o caso brasileiro é ampla no sentido de mostrar que as variáveis relacionadas ao "desenvolvimento regional” são importantes para explicar o desenvolvimento e as disparidades existentes entre as distintas regiões do país (Diniz, 1993; Azzoni, 1997; entre outros).

Dado este problema, a estratégia adotada neste trabalho consiste em, na impossibilidade de trabalhar com um indicador representativo da qualidade das instituições locais (estado) e do "desenvolvimento regional", estabelecer dummies regionais.

\section{RESULTADOS EMPÍRICOS}

A seguir são apresentadas as estimativas obtidas com o auxílio da técnica de regressão quantílica. Como pode ser observado (tabela 1), no primeiro caso a variável explicativa (depvista) tem o sinal esperado e é significativo a $5 \%$ em todos os quantis estimados, mostrando que o desenvolvimento do sistema financeiro afeta positivamente o nível de renda dos estados. Destaca-se, nesse caso, que a magnitude do parâmetro aumenta à 
medida que se aproximam dos quantis superiores da distribuição. As variáveis de controle, em sua grande maioria, mostram ser não significativas, exceção feita à variável que representa os gastos em saúde e saneamento como proporção do PIB, que apresentou sinal negativo e estatisticamente significativo em quatros dos cinco quantis estimados. No segundo caso, a variável deprazo é significante a $1 \%$ em todos os quantis estimados, mostrando novamente que o desenvolvimento do sistema financeiro tem efeitos positivos sobre o nível de renda (dos estados). Por fim, observa-se que a hipótese de convergência de renda não pode ser sustentada a partir das evidências encontradas.

TABELA 1 - RESULTADOS DAS REGRESSÕES QUANTÍLICAS PARA DEPÓSITOS A VISTA E DEPÓSITOS A PRAZO COMO INDICADORES DE DESENVOLVIMENTO DO SISTEMA FINANCEIRO (1995-2004)

\begin{tabular}{|c|c|c|c|c|c|c|c|c|c|c|}
\hline \multicolumn{6}{|c|}{$\begin{array}{c}\text { Var. Dependente: PIB Estadual } \\
\text { Var. Independente: proxy para DF (depvista) }\end{array}$} & \multicolumn{5}{|c|}{$\begin{array}{c}\text { Var. Dependente: PIB Estadual } \\
\text { Var. Independente: proxy para DF (deprazo) }\end{array}$} \\
\hline Quantil & 0,1 & 0,25 & 0,5 & 0,75 & 0,9 & 0,1 & 0,25 & 0,5 & 0,75 & 0,9 \\
\hline Const. & $-0,271$ & $-0,70^{* *}$ & $-0,96^{\otimes}$ & $-0,285$ & $\begin{array}{l}-0,083 \\
\end{array}$ & 0,321 & 0,49 & $0,81 * *$ & $1,26^{*}$ & $1,76^{*}$ \\
\hline renda & $0,911 *$ & $0,832^{*}$ & $0,804^{*}$ & $0,769 *$ & $0,702^{*}$ & $0,88^{*}$ & $0,885^{*}$ & $0,87^{*}$ & $0,84^{*}$ & $0,818^{*}$ \\
\hline educ & $-0,133$ & $0,020 * *$ & $-0,047$ & $-0,012$ & $-0,015$ & $-0,126$ & $-0,122$ & $-0,08^{\otimes}$ & $-0,012$ & 0,035 \\
\hline open & $-0,023$ & $-0,030^{*}$ & $-0,038^{*}$ & $-0,011$ & $-0,006$ & $-0,017$ & $-0,018$ & $-0,012$ & 0,007 & 0,011 \\
\hline educul & $-0,010$ & $-0,006$ & $-0,004$ & $-0,002$ & 0,002 & $-0,009$ & $-0,003$ & $-0,002$ & $-0,004$ & 0,006 \\
\hline sausan & $-0,04^{\otimes}$ & $-0,045^{*}$ & $-0,03^{* *}$ & $-0,03^{*}$ & $-0,023$ & $-0,05^{\otimes}$ & $-0,06 * *$ & $-0,04^{*}$ & $-0,06^{*}$ & $-0,023$ \\
\hline juri & 0,023 & $0,020^{*}$ & 0,0109 & $-0,0005$ & $-0,0005$ & 0,031 & $0,036^{* *}$ & 0,011 & $0,020^{*}$ & $0,016^{* *}$ \\
\hline $\mathrm{DF}$ & $0,09 * *$ & $0,173^{*}$ & $0,206^{*}$ & $0,208^{*}$ & $0,260^{*}$ & $0,074 *$ & $0,069 *$ & $0,068^{*}$ & $0,064 *$ & $0,070^{*}$ \\
\hline$R^{2}$ & 0,93 & 0,92 & 0,92 & 0,92 & 0,92 & 0,93 & 0,92 & 0,91 & 0,91 & 0,91 \\
\hline
\end{tabular}

FONTE: Elaboração dos autores. * significante ao nível de $1 \%$; ** significante ao nível de $5 \% ;{ }^{\otimes}$ significante ao nível de $10 \%$.

NOTA: Os valores de $R^{2}$ referem-se aos valores do pseudo $R^{2}$.

Os resultados para os indicadores de desenvolvimento do sistema financeiro são apresentados a seguir. No primeiro caso (tabela 2), o coeficiente associado ao indicador tamanho de mercado mostrou-se significativo somente no último quantil estimado, onde apresentou o sinal esperado. Deve-se ressaltar que este indicador apresentou sinal negativo para o primeiro quantil, o que, ainda que não significativo, pode sugerir uma relação inversa entre tamanbo de mercado e nível de renda para os estados menos desenvolvidos. Essa relação negativa para o primeiro quantil é confirmada pelo indicador nivel de atividade (1) do sistema bancário, que apresentou coeficientes significativos para o primeiro e último quantil com sinal negativo e positivo, respectivamente. Uma possível explicação para a presença de uma relação inversa nos primeiros quantis, tal como observado anteriormente, advém do fato de que em regiões menos desenvolvidas, onde persiste um ambiente de incerteza crescente, os bancos podem oferecer menos crédito e/ou emprestar menos para essas regiões, dada sua estrutura econômica e o remoto controle sobre as sua filiais; ou ainda, é possível que o sistema financeiro presente nessas regiões atue captando e transferindo recursos dessas para outras 
regiões mais desenvolvidas (menor grau de incerteza), aprofundando assim as desigualdades de renda existentes entre as mesmas.

A estimativa para o segundo indicador do nivel de atividade (2) (tabela 3) exibe um comportamento semelhante aos anteriores. Sendo assim, o coeficiente muda de sinal ao longo da distribuição, sendo negativo para os quantis inferiores e positivo para os superiores. Todavia, este coeficiente mostrou ser significativo somente para o quantis inferiores $(0,10 \mathrm{e}$ 0,25). Dentre as variáveis de controle, destaca-se a variável júri por apresentar coeficiente positivo para três dos cinco quantis estimado. Isso significa que investimentos no sentido de garantir o cumprimento da lei têm impactos positivos sobre o nível de renda, sobretudo, nos estados menos desenvolvidos, onde o ambiente de incerteza é maior.

TABELA 2 - RESULTADOS DAS REGRESSÕES QUANTÍLICAS PARA TAMANHO E NÍVEL (1) DO SISTEMA BANCÁRIO COMO INDICADORES DE DESENVOLVIMENTO DO SISTEMA FINANCEIRO (1995-2004)

\begin{tabular}{|c|c|c|c|c|c|c|c|c|c|c|}
\hline \multicolumn{6}{|c|}{$\begin{array}{c}\text { Var. Dependente: PIB Estadual } \\
\text { Var. Independente: proxy para DF (TamSB) }\end{array}$} & \multicolumn{5}{|c|}{$\begin{array}{c}\text { Var. Dependente: PIB Estadual } \\
\text { Var. Independente: proxy para DF (NivSB1) }\end{array}$} \\
\hline Quantil & 0,1 & 0,25 & 0,5 & 0,75 & 0,9 & 0,1 & 0,25 & 0,5 & 0,75 & 0,9 \\
\hline Const. & 0,057 & 0,246 & $1,14^{*}$ & $1,64^{*}$ & $1,95^{*}$ & $-0,177$ & 0,133 & $0,840^{*}$ & $1,190^{*}$ & $1,10^{*}$ \\
\hline renda & $1,02 *$ & $0,99 *$ & $0,961 *$ & $0,932 *$ & $0,905^{*}$ & $1,03^{*}$ & $1,00^{*}$ & $0,963^{*}$ & $0,937 *$ & $0,930 *$ \\
\hline educ & $-0,09 *$ & $-0,073$ & $-0,12^{\otimes}$ & $-0,032$ & 0,038 & $-0,138$ & $-0,073$ & $-0,127 *$ & $-0,043$ & $-0,010$ \\
\hline open & $-0,038^{*}$ & $-0,03^{* *}$ & $-0,014$ & 0,0018 & 0,003 & $-0,037 *$ & $-0,029 *$ & $-0,015^{* *}$ & $-0,0004$ & $-0,008$ \\
\hline educul & $-0,007^{\otimes}$ & 0,009 & ,0017 & 0,003 & 0,008 & $-0,004$ & 0,002 & 0,003 & 0,004 & $0,011 * *$ \\
\hline sausan & $-0,044^{*}$ & $-0,04 * *$ & $-0,014$ & $-0,03^{*}$ & $-0,016$ & $-0,04 *$ & $-0,037 *$ & $-0,008$ & $-0,02 * *$ & $-0,004$ \\
\hline juri & 0,043 & 0,019 & 0,0018 & $0,023^{*}$ & 0,011 & $0,047^{*}$ & $0,0268 *$ & 0,0001 & 0,0178 & 0,010 \\
\hline $\mathrm{DF}$ & $-0,009$ & 0,030 & 0,017 & 0,026 & $0,067 * *$ & $-0,04 * *$ & $-0,0158$ & 0,0138 & 0,0258 & $0,062^{* *}$ \\
\hline$R^{2}$ & 0,93 & 0,92 & 0,91 & 0,91 & 0,91 & 0,93 & 0,92 & 0,91 & 0,91 & 0,91 \\
\hline
\end{tabular}

FONTE: Elaboração dos autores. * significante ao nível de $1 \%$; $* *$ significante ao nível de $5 \%$; ${ }^{\otimes}$ significante ao nível de $10 \%$.

NOTA: Os valores de $R^{2}$ referem-se aos valores do pseudo $R^{2}$.

TABELA 3 - RESULTADOS DAS REGRESSÕES QUANTÍLICAS PARA NÍVEL (2) E PREFERÊNCIA PELA LIQUIDEZ DO PÚBLICO COMO INDICADORES DE DESENVOLVIMENTO DO SISTEMA FINANCEIRO (1995-2004)

\begin{tabular}{|c|c|c|c|c|c|c|c|c|c|c|}
\hline \multicolumn{6}{|c|}{$\begin{array}{l}\text { Var. Dependente: PIB Estadual } \\
\text { Var. Independente: proxy para DF (NivSB2) }\end{array}$} & \multicolumn{5}{|c|}{$\begin{array}{l}\text { Var. Dependente: PIB Estadual } \\
\text { Var. Independente: proxy para DF (PLP) }\end{array}$} \\
\hline Quantisl & 0,1 & 0,25 & 0,5 & 0,75 & 0,9 & 0,1 & 0,25 & 0,5 & \begin{tabular}{|l|l|} 
& 0,75 \\
\end{tabular} & 0,9 \\
\hline Const. & $-0,173$ & 0,232 & $0,971^{*}$ & $1,22^{*}$ & $1,03^{* *}$ & 0,398 & $0,627 *$ & $1,13^{*}$ & $1,45^{*}$ & $2,25^{*}$ \\
\hline renda & $1,02^{*}$ & $0,999 *$ & $0,962^{*}$ & $0,94^{*}$ & $0,945^{*}$ & $0,968^{*}$ & $0,958^{*}$ & $-0,095^{* *}$ & $0,915^{*}$ & $0,873^{*}$ \\
\hline educ & $-0,12^{* *}$ & $-0,102 *$ & $-0,1^{\otimes}$ & $-0,049$ & $-0,036$ & $-0,17 * *$ & $-0,12^{*}$ & $-0,007$ & $-0,021$ & 0,036 \\
\hline open & $-0,03^{* *}$ & $-0,026$ & $-0,011$ & $-0,0001$ & $-0,010$ & $-0,02^{\otimes}$ & $-0,02 *$ & $-0,0007$ & 0,006 & $0,017^{* *}$ \\
\hline educul & $-0,007$ & $-0,0003$ & 0,0013 & $0,007^{* *}$ & $0,011^{\otimes}$ & $-0,01$ & $-0,005$ & $-0,02 * *$ & $-0,0001$ & $0,009^{\otimes}$ \\
\hline sausan & $-0,049 *$ & $-0,033^{*}$ & $-0,007$ & $-0,02 * *$ & $-0,005$ & $-0,06 * *$ & $-0,049 *$ & 0,0005 & $-0,055^{*}$ & $-0,017$ \\
\hline juri & $0,0533 *$ & $0,027 *$ & 0,0012 & $0,019 * *$ & 0,004 & $0,034^{\otimes}$ & $0,027^{*}$ & $-0,023$ & $0,024 *$ & $0,019 *$ \\
\hline $\mathrm{DF}$ & $-0,069 *$ & $-0,032^{* *}$ & $-0,025$ & 0,008 & 0,0227 & $-0,118^{*}$ & $-0,099 *$ & $-0,073^{*}$ & $-0,070^{*}$ & $-0,113^{*}$ \\
\hline$R^{2}$ & 0,93 & 0,92 & 0,91 & 0,91 & 0,90 & 0,93 & 0,92 & 0,91 & 0,914 & 0,91 \\
\hline
\end{tabular}

FONTE: Elaboração dos autores. * significante ao nível de $1 \%$; ** significante ao nível de $5 \%$; ${ }^{\otimes}$ significante ao nível de $10 \%$.

NOTA: Os valores de $R^{2}$ referem-se aos valores do pseudo $R^{2}$. 
A tabela 3 também apresenta os resultados do exercício econométrico que busca captar o argumento pós-keynesiano em relação ao impacto da preferência pela liquidez do público sobre o nível de renda. Para tanto, seguiu-se a mesma metodologia anterior. A hipótese teórica é que quanto maior for PLP, menor será a oferta de crédito e, portanto, menor será o crescimento econômico. Assim, em termos econométricos, os coeficientes esperados devem apresentar sinal negativo e devem exibir magnitude decrescente dos primeiros para os últimos percentis.

Como pode ser observado, o indicador de preferência pela liquidez do público exibe o comportamento esperado, uma vez que o sinal dos coeficientes foi negativo e estatisticamente significativo a 1\%, sendo que, em magnitude, os mesmos exibiram o comportamento decrescente esperado, exceção feita ao último quantil.

\section{CONSIDERAÇÕES FINAIS}

Este trabalho desenvolveu uma análise empírica com o objetivo de estudar a relação entre desenvolvimento financeiro e crescimento econômico para o caso brasileiro. Sendo assim, utilizou-se a técnica de regressão quantílica para dados das unidades federadas do Brasil e as evidências encontradas sugerem a existência de uma relação positiva.

Para mensurar o desenvolvimento do sistema financeiro adotaram-se duas estratégias: a primeira segue o trabalho de King \& Levine (1993), que define algumas variáveis financeiras como indicadores de desenvolvimento desse sistema. Nesse caso, a relação encontrada foi positiva para ambos os indicadores. Por outro lado, foram construídos alguns indicadores para tamanho e nível de atividade do sistema financeiro. As evidências encontradas tendem a confirmar a relação anterior, embora deve-se ressaltar que estes últimos indicadores mostraram existir uma relação negativa entre o desenvolvimento do sistema financeiro e o nível de renda para os quantis inferiores, sugerindo uma não funcionalidade desse sistema, o que tende a agravar as disparidades de renda regional.

\section{REFERÊNCIAS}

ACEMOGLU, D. Introduction to modern economic growth. Princeton: Princeton University Press, 2009.

AZZONI, C. R. Concentração regional e dispersão das rendas per capita estaduais: análise a partir de séries históricas estaduais de PIB, 1939-1995. Estudos Econômicos, São Paulo, v. 30, n. 2, p. 207-231, abr./jun. 1997. 
BARRO, R. Health and economic growth. Cambridge, MA: Harvard University, 1996. Mimeo.

BECK, T.; LEVINE, R.; LOAYZA, N. Finance and sources of growth. Journal of Financial Economics, Rochester, Nova Iorque, vol. 58, pp. 261-300, 2000.

BLOOM, D.E; CANNING, D.; SEVILLA, J. The effect of health on economic growth: theory and evidence. NBER Working Paper No. w8587, 2001.

CAVALCANTE, A.; CROCCO, M.; JAYME JR., F. Preferência pela liquidez, sistema bancário e disponibilidade de crédito regional. In: CROCCO, M.; JAYME JR., F. Moeda e território: uma interpretação da dinâmica regional brasileira. Belo Horizonte: Autêntica, 2006. p.295-315.

DINIZ, C. Desenvolvimento poligonal no Brasil: nem desconcentração nem contínua polarização. Revista Nova Economia, Belo Horizonte, v. 3, n. 1, 1993.

KING, R.; LEVINE, R. Financial intermediation and economic development. In: MAYER, C.; VIVES, X. Financial Intermediation in the Construction of Europe, Londres: Center for Economic Policy Research, 1993a, p. 156-189.

LEVINE, R. Financial development and economic growth: views and agenda. Journal of Economic Literature, v. 35, pp. 688-726, 1997.

LEVINE, R.; LOAYZA, N.; BECK, T. Financial intermediation and growth: causality and causes. Journal of Monetary Economics, v. 46, p. 31-77, 2000.

ROUSSEAU, P. L.; WACHTEL, P. Financial intermediation and economic performance: historical evidence from five industrialized countries. Journal of Money, Credit, and Banking, v. 30, n. 4, p. 657-678, 1998.

SCHUMPETER, J. The theory of economic development. Cambridge, MA: Harvard University Press, 1911. 
